\title{
ADAPTACIÓN Y VALIDEZ DE CONTENIDO DEL CUESTIONARIO DE ENVIDIA INFANTIL A POBLACIÓN ADULTA ADAPTATION AND CONTENT VALIDITY OF THE CHILD ENVY QUESTIONNAIRE TO ADULT POPULATION
}

\author{
Enrique Vila Abad, Luis Alfonso Hijós Larraz y Francisco Pablo Holgado Tello \\ Universidad Nacional de Educación a Distancia (UNED)
}

Cómo referenciar este artículo/How to reference this article:

Vila Abad, E., Hijós Larraz, L. A. y Holgado-Tello, F. P. (2013). Adaptación y Validez de Contenido del Cuestionario de Envidia Infantil a Población Adulta [Adaptation and Content Validity of the Child Envy Questionnaire to Adult Population]. Acción Psicológica, 10(2), 59-72. http://dx.doi.org/10.5944/ ap.10.2.11824

\section{Resumen}

En este trabajo se define la envidia como una emoción de particular complejidad, de carácter displacentero, caracterizado por sentimientos de inferioridad, hostilidad y resentimiento (relacionado con la percepción de injusticia), producido por la toma de conciencia de que otra persona o grupo de personas disfrutan de una posesión deseada (objeto, posición social, atributo). El objetivo de esta investigación ha sido explorar la envidia en la población adulta. Para ello, se partió de un instrumento autoinformado de fácil aplicación, basado en el Cuestionario de la Envidia Infantil (CEI) desarrollado por Carrasco et al (2004). Para ello, después de adaptar el contenido morfosemantico de los ítems, se realizó un juicio de expertos en el que participaron 12 jueces que analizaron la relevancia y representatividad del pool inicial de ítems. El acuerdo entrejueces se midió mediante el índice de Osterlind, seleccionando aque- llos con mayor congruencia $\left(\mathrm{I}_{\mathrm{ik}} \geq 0.5\right)$ para constituir la versión del cuestionario de envidia en adultos (CEA) con 24 ítems.

Palabras clave: adultos, envidia, validez contenido, juicio expertos..

\section{Abstract}

In this study envy is defined as an emotion of particular complexity, unpleasant character, characterized by feelings of inferiority, hostility and resentment (related to the perception of injustice), produced by the realization that another person or group of persons enjoy a desired possession (subject, status, attribute). The objective of this research was to explore the envy of the adult population. To do this, we adapted an instrument easy to apply self-reported, based on the CIS developed by Carrasco, Gonzalez \& Barrio (2004) and as a first step was to get evidence of content validity. After

Correspondencia: Enrique Vila Abad. Universidad Nacional de Educación a Distancia (UNED). Facultad de Psicología, C/Juan del Rosal 10. 28040 Madrid. Email: evila@psi.uned.es 
adapting the content of the items morphosemantical held an expert opinion for the analysis of content validity, using the index of Osterlind, selecting those with greater consistency $\left(\mathrm{I}_{\mathrm{ik}} \geq 0.5\right)$ to form the final version of the questionnaire envy in adults (CEA) with 24 items representing adequately all the dimensions. In conclusion, the CEA is a suitable instrument for assessing the envy of a valid and reliable way, being composed of 24 items representing all theoretical dimensions proposed.

Keywords: Adult, envy, content validity, expert judgment.

\section{Introducción}

Nadie pone en duda el papel de las emociones para modular y motivar la conducta, llegando a actuar como enlace entre el organismo y el entorno (Riedl, 2005). Algunas emociones -primarias- emergen en los primeros momentos de la vida cumpliendo una función adaptativa, pero otras-secundarias- aparecen dentro del complejo proceso de socialización influyendo en el desarrollo de capacidades cognitivas (Fernández-Abascal, Jiménez y Martín Díaz, 2007). Éstas se consideran también sociales o autoconscientes ya que surgen de la relación con los demás, aparecen en un contexto social determinado, que es el que les confiere significación, y necesitan del análisis de los otros para poder entenderse apropiadamente (Etxebarria, 2008; Sawada, 2010; Tangney y Salovey, 2010). Estas experiencias emocionales son complejas, ya que se encuentran compuestas de otras más sencillas, más elementales, y que lejos de tratarse de emociones simples, pueden adquirir una intensidad incluso más elevada que cualquier emoción básica e implicar conductas de un alcance bastante mayor (Choliz y Gomez, 2002).

Entre estas emociones secundarias, derivadas, complejas, sociales y morales dentro de un grupo más amplio (se incluyen la vergüenza, celos, indignación...) se encuentra la envidia, la cual se caracteriza por las negativas reacciones afectivas ante la comparación social produciendo ciertas consecuencias negativas, o, desadap- tativas (Etxebarria, 2008; Riedl, 2005; Smith y Kim, 2007; Tangney y Salovey, 2010).

Muchos autores consideran la envidia como una emoción desagradable que puede surgir cuando se compara un individuo con los demás; es una experiencia común para la mayor parte de las personas independiente de la cultura a la cual se pertenezca. La envidia se caracteriza por sentimientos de inferioridad, hostilidad y resentimiento (relacionado con la percepción de injusticia) producido por la toma de conciencia de que otra persona o grupo de personas disfrutan de una posesión deseada -objeto, posición social, atributo(Smith y Kim, 2007). Tal como recuerdan Milfont y Gouveia (2009), hay dos componentes principales en la envidia: hostilidad y depresión. El componente hostil se asocia con los sentimientos de rencor y rabia que se derivan de creencias subjetivas de injusticia. El componente depresivo se asocia con sentimientos de inferioridad, que se derivan de la comparación social desfavorable. Parrot y Smith (1993) describen seis tipos de episodios emocionales que suelen formar parte de la envidia: deseo de lo que tiene la otra persona, hostilidad y admiración hacia ella, resentimiento global, sentimientos de inferioridad y de culpa.

Así, la envidia es un afecto de particular complejidad, de carácter displacentero, vinculado a nuestras autoevaluaciones en atributos que se consideran relevantes, que determinan una sensación de falta o limitación y por tanto, deviene un sentimiento de inferioridad (Salovey, 1991). Se presenta cuando la persona envidiada tiene similares características con la envidiosa, éstas son relevantes para la comparación, el dominio de comparación es importante para la persona envidiosa y las perspectivas de equiparación con la persona enviada no es probable, a pesar de la similitud entre ambas (Smith y Kim, 2007).

Al ser una emoción con repercusiones negativas ha sido objeto de conocimiento de los seres humanos desde puntos dispares tanto religiosos como filosóficos o literarios (Riedl, 2005). Dentro de la literatura científica, la envidia ha ocupado un papel central en la antropología y en la sociología destacando como 
punto álgido el desarrollo de Schoeck (1987) en su libro "Envy: A Theory of Social Behaviour», que han dado lugar a que actualmente se emprendan estudios transculturales sobre los diferentes tipos de envidia (van de Ven, Zeelenberg y Pieters, 2009) o los estudios antropológicos sobre la importancia de la envidia en la conducta social (Izquierdo y Johnson, 2007).

La psicología ignoró la envidia al igual que otras emociones por su carácter subjetivo, considerándose por lo tanto inaccesible para la investigación y no sistematizable. A ello ha contribuido la elevada influencia de la deseabilidad social sobre cualquier intento de estudio de la envidia a través de los instrumentos de uso más frecuente en psicología como son los cuestionarios e inventarios. Solamente la tradición psicoanalista se seguía ocupando de la envidia, dándole un papel importante (envidia del pene, del vientre y del pecho). Actualmente, los psicoanalistas dan importancia a los estudios de la envidia incidiendo en aspectos relativos a su génesis (Anderson 2011; Bonovitz 2010; Debbané 2011; Etchegoyen y Nemas, 2003; Laverde-Rubio, 2004; Rosenberger, 2005; Winarick, 2010; West 2010; o a su relación con trastornos de la personalidad (Hirsch, 2011; Krizan y Johar, en prensa), o la diferenciación de diferentes tipos de envidia (Shoshani, Shoshani, Kella y Becker, 2011). Los estudios anteriores son contemporáneos con la búsqueda de bases neuroanatómicas de la envidia, (Shamay-Tsoory, Tibi-Elhanany y Aharon-Peretz, 2007; Takahashi, 2009; Krajbich, Adolphs, Tranel, Denburg y Camerer 2009; Dvash, Gilam, Ben-Ze'ev, Hendler y Shamay-Tsoory, 2010), o la implicacion de neurotransmisores en dicha emoción (Shamay-Tsoory, Fischer, Dvash, Harari, Perach-Bloom y Levkovitz, 2009).

Pero el avance importante de los estudios de la envidia se ha realizado dentro de la psicología social, produciéndose tras el desarrollo del concepto de comparación social de Festinger (1954), que ha servido como marco de trabajo para el estudio de la misma. La envidia surge en la interacción humana y se expresa en ella (Salovey, 1991). En la actualidad, partiendo de la comparación social, existe un abordaje desde los diferentes puntos de vista de las ciencias cognitivas, dando lugar a que estudien distintos aspectos de la envidia como su papel adaptativo (Coricelli y Rsutichini, 2010), la influencia del autocontrol (Crusius y Mussweiler, 2011; Hill, DelPriore y Vaughan, 2010), de la autoevaluación, de la autoestima (van de Ven, Zeelenberg y Pieters, 2010; van Dijk, Ouwerkerk, Wesseling y van Koningsbruggen, 2011), de la percepción de injusticia (Cohen-Charash y Mueller, 2007; Johar et al., 2011) en el desarrollo de la envidia, o la relacion de la envidia con otras emociones (van de Ven, Zeelenberg y Pieters, 2011).

Actualmente no se duda que la envidia afecta al estado fisiológico, biológico, psicológico, interpersonal, social, etc., lo que refleja la importancia en la comprensión de esta emoción para la explicación del comportamiento humano (Riedl, 2005). Las acciones hostiles o agresivas que pueden dar lugar a conductas criminales nos apuntan en dicha dirección, así como la relación de la envidia con el trastorno de la personalidad narcisista (Hirsch, 2011; Krizan y Johar, 2012), o las consecuencias sobre la atención y la memoria (Hill et al., 2010), igualmente la existencia de una relación negativa entre la envidia y el bienestar, ya que las personas con una disposición envidiosa tienen más probabilidades de ser infelices y de estar insatisfechos con sus vidas, además de tener sentimientos de baja vitalidad y energía. Por lo tanto, la tendencia a la envidia debería reducirse si se busca el bienestar (Milfort y Gouvenia, 2009). Este resultado se encuentra dentro de la línea de aquellos autores que afirman que las emociones positivas potencian la salud, mientras que las emociones negativas tienden a disminuirla (Fernández-Abascal y Palmero, 1999). Se han realizado estudios sobre la envidia en distintos ámbitos e instituciones, incluyendo su influencia de la envidia en actividades educativas (Heikkinen y Isola, 2004; Heikkinen, Latvala y Isola, 2003), laborales (Kragl y Schmid, 2010; Leon 2002; Tai, Narayanan y McAllister, 2012), o deportivas (McNamee, 2010).

Para la valoración de la envidia se han utilizado evaluaciones directas e indirectas; estas últimas se usaron por el carácter de emoción no exhibible de la envidia (Habimana y Massé, 2000), como por ejemplo las que se han llevado 
a cabo mediante el uso de viñetas que consisten en pequeñas historias o cuentos de situaciones o eventos emocionales (Reidl, 2005) o mediante el rendimiento en una tarea (Kragl y Schmid, 2010). También se han utilizado medidas biofisiológicas (Bediou, Mohri, Lack y Sander 2011; Fiske 2010) e incluso se han analizado aspectos no verbales (Thompson y Hampton 2011).

Entre las medidas directas señalar el uso de autoinformes o de relatos emocionales (Bediou et al., 2011; Krizan y Johar, 2011; Rodriguez, Parrott y Hurtado, 2010).

De una manera mas genérica, se encuentra el cuestionario desarrollado por Gold (1996) que utiliza ítems que contienen expresiones familiares, resultando un método fiable y cuyos resultados correlacionan con otras medidas sugiriendo una adecuada validez de criterio; con la Social Comparisons Inventory (Masse et al., 1996) de los que se han obtenido 4 factores relativos a contenidos de popularidad, bienestar financiero, bienestar personal, inteligencia y talento; y el Dispositional Envy Scale (DES; Smith et al., 1999), existiendo su versión para niños con similares propiedades psicométricas Dispositional Envy Scale for Children (DESC; Sawada y Arai, 2002). Este cuestionario consta de 8 ítems, cuatro de los cuales preguntan a los entrevistados el grado y la frecuencia de sus experiencias de la envidia. La estructura factorial de la envidia en este cuestionario es unidimensional (Milfort y Gouvenia, 2009), no estando validado para población española.

Sin olvidar que los cuestionarios e inventarios también pueden presentar problemas como el auto-alabarse, los prejuicios defensivos, la incertidumbre acerca de la interpretación que los sujetos hacen de las preguntas, las predisposiciones de respuesta tales como la deseabilidad social, la aquiescencia o el uso de respuestas extremas y la codificación incompleta del evento (Rield, 2005); la escala DES y las de ella derivadas se encuentran además con que el reconocimiento explícito de la emoción analizada lo que provoca que se tienda a ocultarla por la naturaleza indeseable de la envidia, por lo que probablemente no se capten todos los aspectos de la misma. Especialmente, los instrumentos deberían evitar ítems que contengan la palabra envidia ya que las personas que la admiten no aceptan todos los sentimientos que esta conlleva, evitando aquellos items con gran carga social especialmente los relacionados con la hostilidad y el resentimiento (Smith y Kim, 2007).

Entre los instrumentos que no presentan este problema se encuentra el Cuestionario de envidia infantil (CEI) desarrollado por Carrasco et al (2004), desarrollado con la finalidad de poder medir de una forma fácil la envidia en la población infantil española. La versión definitiva de este cuestionario consta de 21 ítems, cada uno de ellos con cinco opciones de respuesta. Se obtuvieron adecuados valores de fiabilidad y validez, y en el análisis factorial confirmatorio se identifica una estructura bifactorial de la envidia, compuesta por un primer factor relacionado con la reacción envidiosa que incluye los sentimientos negativos ante el logro o la admiración de los otros, la degradación de la posición social o la imagen del envidiado, y los pensamientos de injusticia ante los logros y adquisiciones de los demás y un segundo factor, de deseo por lo ajeno, siendo coincidentes con los elementos definitorios descritos por Castilla del Pino (2000). González, Carrasco y del Barrio (2011) han estudiado con este instrumento las relaciones de la envidia con otras emociones y las diferencias por edad y sexo.

Las aplicaciones y direcciones futuras de la investigación en el campo de la envidia van encaminadas al conocimiento de las relaciones intergrupales y al estudio del prejuicio envidioso, las relaciones entre la envidia y la salud (psíquica y física), y los tipos de estrategias de afrontamiento de la envidia (Smith y Kim, 2007). Así, se hace necesario el desarrollo de instrumentos adecuados para la medición de la envidia.

El objetivo general de este trabajo es explorar la envidia en población adulta. Para ello, y debido a los pocos instrumentos psicometricos adaptados y validados en nuestro medio cultural, se marcan como objetivos específicos la adaptación a la población adulta del Cuestionario de la envidia infantil utilizado como punto de partida los ítems utilizando por Carrasco et 
al. (2004) y la obtención de evidencias de validez de contenido de las misma, con el fin de obtener un instrumento autoinformado de fácil aplicación para la evaluación de la envidia.

\section{Metodología}

\section{Participantes}

Para el estudio de validación de contenido se contó con la participación de una muestra compuesta por expertos en evaluación y/o medida, tanto del ámbito académico (facultad de psicología de la UNED y facultad de pedagogía de la Universidad de Zaragoza) como de la psicoterapia. Por lo tanto, el muestreo fue intencional. La muestra estuvo compuesta por un total de 12 psicólogos, seis jueces relacionados con el área de enseñanza e investigación y los otros seis relacionados con la actividad psicoterapéutica.

\section{Instrumentos}

Los instrumentos utilizados para la realización de este trabajo fueron:

El pool inicial de items adaptado semánticamente a población adulta utilizado para el desarrollo del CEI (Carrasco et al (2004). Se evalúan las siguientes dimensiones: (a) Sentimientos negativos cuando se elogia a alguien que se admira o que tiene algo que se desea: ítems $1,6,11$, 16, 21, 26, 31; (b) Degradación de la posición social o la imagen del envidiado: ítems $2,3,7,8$, 12, 17, 22, 32, 36, 37, 41, 42, 45, 46; (c) Desear cosas que tienen los demás: ítems 13, 18, 21, 23, 28, 33, 38, 43, 47; (d) Pensamientos de injusticia ante los logros y adquisiciones de los demás: ítems 4, 9, 14, 19, 24, 29; (e) Sentimientos negativos ante la adquisición de logros de los demás: ítems 34, 35, 39, 40, 44, 48; y (f) Relación con el envidiado: 5, 10, 15, 20, 25, 27, 30.

Protocolo para la obtención de evidencias de validez de contenido. Para la valoración de las dimensiones utilizadas en el CEI se diseñó un cuestionario para la cumplimentación por los expertos que evaluaba el grado de «idoneidadcongruencia» en la asignación de los distintos indicadores a las dimensiones de la envidia planteadas.

La escala que se utilizó para valorar los indicadores, fue la siguiente: $A$ (alto grado de idoneidad); $M$ (medio grado de idoneidad); y $B$ (bajo grado de idoneidad).

Para la valoración de las respuestas de los jueces se calcula el índice de congruencia de Osterlind (1989) para cada indicador, de acuerdo con la ecuación 1.

$$
I_{i k}=\frac{(\mathrm{N}-1) \sum_{j=1}^{n} \mathrm{X}_{i j k}+\mathrm{N} \sum_{j=1}^{n} \mathrm{X}_{i j k}-\sum_{j=1}^{n} \mathrm{X}_{i j k}}{2(\mathrm{~N}-1) \mathrm{n}}
$$

$X_{\mathrm{ijk}}=$ Valoración del indicador $i$ en el dominio $k$ por el juez $j$.

$\mathrm{N}$ = Número de dominios que se contemplen en el instrumento.

$\mathrm{n}=$ Número de jueces que valoran el indicador.

Se consideró un nivel de congruencia adecuado aquel con una puntuación obtenida en este índice mayor o igual a $0.5\left(\mathrm{I}_{\mathrm{ik}} \geq 0.5\right)$.

\section{Procedimiento}

En primer lugar se revisó la redacción de los ítems utilizados para la construcción del CEI, para adaptar su contenido y redacción a población adulta. Se contó con la colaboración de 17 estudiantes del Campus universitario de Huesca, que después de realizar una valoración morfosemántica aportaron sus oportunas correcciones. Seguidamente se realizó la primera prueba del mismo, para conocer las condiciones de aplicación, con el objetivo de detectar ítems con problemas de comprensión debidos a una mala redacción.

Posteriormente se rectificaron términos que, en alguno de los elementos, mostraron en esta aplicación confusión o dificultades de comprensión. Para finalizar con esta fase preliminar se diseñó el formato de la prueba, que se compuso de 48 ítems adaptados a adultos 
que representan las dimensiones indicadas. Así mismo, se establecieron unas breves instrucciones para los sujetos, estimándose un tiempo de realización no superior a una hora.

A continuación, para obtener evidencias de validez de contenido de los ítems se realizó el juicio de expertos, que permitió eliminar aquellos ítems que no cumplían los requisitos adecuados, para lo cual se valoraron las dimensiones utilizadas en el diseño del CEI. Se elaboraron unas breves instrucciones relacionadas con el protocolo para la obtención de evidencias de validez de contenido.

Se procedió a la selección de los indicadores con mayor puntuación $\left(\mathrm{I}_{\mathrm{ik}} \geq 0.5\right)$ en el índice de Osterlind, para formar parte del sistema de indicadores.

\section{Resultados}

A continuación se muestran una serie de tablas en las que se presentan los indicadores más representativos de las dimensiones particulares de cada una de dichas áreas. Cada tabla contiene en las primeras columnas el número de ítem y la denominación del ítem. Una tercera columna donde aparece el valor del índice de Osterlind. En la siguiente columna aparecen los comentarios de los jueces y para concluir se muestra una columna donde aparece el número de expertos que valoraron el item. Se presentan en negrilla los indicadores que han obtenido una mejor puntuación en cuanto a su grado de idoneidad $\left(\mathrm{I}_{\mathrm{ik}}>0.5\right)$, y que por tanto fueron seleccionados entre los ítems propuestos para continuar dentro del proceso de selección.

En la tabla 1, respecto a la dimensión A (Sentimientos negativos cuando se elogia a alguien que se admira o que tiene algo que se desea), se encuentran dos ítems con un amplio consenso $\left(\mathrm{I}_{\mathrm{ik}}=0.92\right)$ sin que existan comentarios de los jueces.

Tabla 1

Dimensión A: Sentimientos negativos cuando se elogia a alguien que se admira o que tiene algo que se desea

\begin{tabular}{|c|c|c|c|c|}
\hline N. ${ }^{\circ}$ ítem & Enunciado & $I_{i k}$ & Comentarios de los expertos & $\mathbf{N}$ \\
\hline ítem 11 & $\begin{array}{l}\text { Me siento mal cuando alguien } \\
\text { reconoce las cualidades y lo bueno } \\
\text { de mis compañeros. }\end{array}$ & 0.92 & & 12 \\
\hline ítem 16 & $\begin{array}{l}\text { Me molesta que mis compañeros } \\
\text { reciban elogios como yo querría para } \\
\text { mí. }\end{array}$ & 0.92 & & 12 \\
\hline ítem 1 & $\begin{array}{l}\text { Me mantengo en silencio cuando } \\
\text { hablan bien de un compañero. }\end{array}$ & 0.00 & $\begin{array}{l}\text { Ítem con componente adicional en } \\
\text { términos de competencia social o de } \\
\text { reticencia. }\end{array}$ & 12 \\
\hline ítem 26 & $\begin{array}{l}\text { Me gustan las personas que destacan } \\
\text { en un grupo. }\end{array}$ & -0.25 & $\begin{array}{l}\text { Agregar que destacan por cosas } \\
\text { positivas, méritos, habilidades. }\end{array}$ & 12 \\
\hline ítem 6 & $\begin{array}{l}\text { Me agrada que se reconozcan las } \\
\text { buenas cualidades de mis compañeros. }\end{array}$ & -0.25 & & 12 \\
\hline ítem 31 & $\begin{array}{l}\text { Me agrada cuando un compañero hace } \\
\text { bien su trabajo. }\end{array}$ & -0.33 & & 12 \\
\hline
\end{tabular}

En la dimensión B (tabla 2) se aprecia que han sido seleccionados ocho ítems entre los que destaca el consenso absoluto del ítem 36.
Respecto a los comentarios de los jueces reseñar la existencia de solapamientos entre los ítems. 
Tabla 2

Dimensión B: Degradación de la posición social o la imagen del envidiado

\begin{tabular}{|c|c|c|c|c|}
\hline N. ${ }^{\circ}$ ítem & Enunciado & $\mathrm{I}_{\mathrm{ik}}$ & Comentarios de los expertos & $\mathbf{N}$ \\
\hline ítem 36 & $\begin{array}{l}\text { Hablo mal de las personas que } \\
\text { tienen cosas que a mí me gustaría } \\
\text { tener }\end{array}$ & 1.00 & $\begin{array}{l}\text { Solapamiento con el ítem } 46 \text {. Se podría } \\
\text { añadir la posibilidad de «difamación del } \\
\text { envidiado». }\end{array}$ & 12 \\
\hline ítem 41 & $\begin{array}{l}\text { Me gusta hacer daño a los que } \\
\text { consiguen cosas que yo no he } \\
\text { podido conseguir }\end{array}$ & 0.92 & & 12 \\
\hline ítem 42 & $\begin{array}{l}\text { Si un compañero recibe una } \\
\text { recompensa o distinción, lo } \\
\text { menosprecio }\end{array}$ & 0.92 & & 12 \\
\hline ítem 17 & $\begin{array}{l}\text { Le quito importancia a los meritos } \\
\text { que consiguen mis compañeros }\end{array}$ & 0.83 & & 12 \\
\hline ítem 37 & $\begin{array}{l}\text { Desprecio a los que consiguen } \\
\text { cosas que yo quiero }\end{array}$ & 0.83 & & 12 \\
\hline ítem 32 & $\begin{array}{l}\text { Cuando un compañero obtiene } \\
\text { buenos resultados no ha sido por } \\
\text { meritos propios. }\end{array}$ & 0.75 & Solapamiento con ítem 9. & 12 \\
\hline ítem 8 & $\begin{array}{l}\text { Si un compañero tiene éxito suelo } \\
\text { decir que ha sido suerte }\end{array}$ & 0.58 & $\begin{array}{l}\text { Solapamiento con el 33. La redacción } \\
\text { sería mejor si se escribe: suelo decir que } \\
\text { ha sido debido a la suerte }\end{array}$ & 12 \\
\hline ítem 45 & $\begin{array}{l}\text { Comento principalmente cosas } \\
\text { negativas de mis compañeros }\end{array}$ & 0.50 & $\begin{array}{l}\text { La redacción sería mejor si se escribe: } \\
\text { Intento disminuir la imagen positiva de } \\
\text { algún compañero incluso recurriendo a la } \\
\text { mentira. }\end{array}$ & 12 \\
\hline ítem 46 & $\begin{array}{l}\text { Las recompensas y premios que } \\
\text { reciben mis compañeros suelen ser de } \\
\text { poca importancia }\end{array}$ & 0.33 & & 12 \\
\hline ítem 2 & $\begin{array}{l}\text { Le doy mucha importancia a las } \\
\text { virtudes que tienen mis compañeros }\end{array}$ & -0.08 & $\begin{array}{l}\text { Se puede dar mucha importancia a las } \\
\text { virtudes de los otros porque siempre se } \\
\text { intenta «ver» lo positivo de los demás (lo } \\
\text { cual sería una costumbre muy positiva y } \\
\text { alejada de la envidia) o porque se focaliza } \\
\text { la mirada en nuestras carencias y que no } \\
\text { podemos soportar que otras personas } \\
\text { posean (envidia). }\end{array}$ & 12 \\
\hline ítem 22 & $\begin{array}{l}\text { Me resulta fácil reconocer lo bueno que } \\
\text { tienen mis compañeros }\end{array}$ & -0.17 & & 12 \\
\hline ítem 12 & $\begin{array}{l}\text { Reconozco fácilmente lo bueno que } \\
\text { tienen mis compañeros }\end{array}$ & -0.25 & & 12 \\
\hline ítem 3 & $\begin{array}{l}\text { Considero que ni el éxito ni el dinero } \\
\text { da la felicidad }\end{array}$ & -0.42 & & 12 \\
\hline ítem 7 & Hablo bien de mis compañeros. & -0.50 & $\begin{array}{l}\text { ítem que puede tener otro componente } \\
\text { adicional en términos de competencia } \\
\text { social o de reticencia }\end{array}$ & 12 \\
\hline
\end{tabular}


En la tabla 3 se exponen los resultados de la dimensión $\mathrm{C}$, relativa a desear cosas que tienen los demás; en ella se observa que los cuatro ítems seleccionados tiene el valor de consenso mínimo para continuar en la selección.

Tabla 3

Dimensión C: Desear cosas que tienen los demás

\begin{tabular}{|c|c|c|c|c|}
\hline N.․ ítem & Enunciado & $I_{i k}$ & Comentarios de los expertos & $\mathbf{N}$ \\
\hline ítem 21 & $\begin{array}{l}\text { Deseo tener las cosas que tienen los } \\
\text { demás }\end{array}$ & 0.50 & «algunas cosas» & 12 \\
\hline ítem 33 & $\begin{array}{l}\text { Creo que merezco las cosas buenas } \\
\text { que tienen los demás }\end{array}$ & 0.50 & & 12 \\
\hline ítem 38 & $\begin{array}{l}\text { Querría tener el nivel de vida y los } \\
\text { recursos económicos que tienen los } \\
\text { demás }\end{array}$ & 0,50 & & 12 \\
\hline ítem 43 & $\begin{array}{l}\text { Me gustaría que todos mis } \\
\text { compañeros quisieran estar más } \\
\text { conmigo que con los otros }\end{array}$ & 0.50 & & 12 \\
\hline ítem 28 & $\begin{array}{l}\text { Quisiera recibir los elogios que reciben } \\
\text { alguno de mis compañeros }\end{array}$ & 0.00 & & 12 \\
\hline ítem 13 & $\begin{array}{l}\text { Quisiera tener algunas cosas que } \\
\text { tienen los demás. }\end{array}$ & -0.08 & $\begin{array}{l}\text { El deseo de tener «algo» que los demás } \\
\text { poseen puede convertirse en admiración, } \\
\text { convirtiéndose la persona en un modelo a } \\
\text { seguir y no en un objeto de envidia. }\end{array}$ & 12 \\
\hline ítem 23 & $\begin{array}{l}\text { Quisiera tener tan buenos resultados } \\
\text { como algunos compañeros }\end{array}$ & -0.17 & Ítem indicativo de autoestima & 12 \\
\hline ítem 47 & $\begin{array}{l}\text { Quisiera ser el que decidiera a dónde } \\
\text { ir o a qué hacer cuando salgo con mis } \\
\text { amigos }\end{array}$ & -0.17 & $\begin{array}{l}\text { Item con alto componente de } \\
\text { competencia social }\end{array}$ & 12 \\
\hline ítem 18 & $\begin{array}{l}\text { Desearía ser tan bueno en el trabajo } \\
\text { (estudio) como los demás. }\end{array}$ & -0.25 & $\begin{array}{l}\text { ítem indicativo de autoconcepto y } \\
\text { autoestima }\end{array}$ & 12 \\
\hline
\end{tabular}

En la tabla 4 aparecen los datos relativos a los pensamientos de injusticia ante logros y adquisiciones de los demás. Para la medida de esta dimensión fueron seleccionados 5 ítems, encontrando un gran valor de consenso en el ítem 24. Los cambios de redacción fueron propuestos por uno de los jueces. 
Tabla 4

Dimensión D: Pensamientos de injusticia ante los logros de los demás

\begin{tabular}{|c|c|c|c|c|}
\hline N. ${ }^{\circ}$ ítem & Enunciado & $I_{\mathrm{ik}}$ & Comentarios de los expertos & $\mathbf{N}$ \\
\hline ítem 24 & $\begin{array}{l}\text { Cuando alguien me supera suelo } \\
\text { pensar que es injusto }\end{array}$ & 0.92 & $\begin{array}{l}\text { También se podría redactar así: Tengo } \\
\text { más atributos que los demás, pero no se } \\
\text { me reconocen (el envidioso suele afirmar } \\
\text { que él posee muchos más atributos y } \\
\text { habilidades que los demás, pero que } \\
\text { injustificadamente no se le valoran.) }\end{array}$ & 12 \\
\hline ítem 4 & $\begin{array}{l}\text { Pienso que es injusto que los demás } \\
\text { tengan cosas que yo no tengo }\end{array}$ & 0.75 & $\begin{array}{l}\text { Agregaría: cosas que yo no tengo y } \\
\text { deseo }\end{array}$ & 12 \\
\hline ítem 9 & $\begin{array}{l}\text { Los demás no deberían conseguir lo } \\
\text { que yo no puedo tener }\end{array}$ & 0.67 & $\begin{array}{l}\text { Se podría redactar así: Los demás no } \\
\text { deberían conseguir las cosas que deseo } \\
\text { y no logro tener. }\end{array}$ & 12 \\
\hline ítem 19 & Nadie debería ser mejor que yo. & 0.58 & $\begin{array}{l}\text { ítem con un posible componente de alta } \\
\text { deseabilidad social }\end{array}$ & 12 \\
\hline ítem 29 & Si pierdo pienso que no es justo & 0.50 & & 12 \\
\hline ítem 14 & $\begin{array}{l}\text { Es injusto que existan personas que } \\
\text { tengan más dinero que yo }\end{array}$ & 0.42 & $\begin{array}{l}\text { Sería oportuno especificar a las } \\
\text { «personas». En lugar de ser tan } \\
\text { genéricas, podría centrarse en grupos } \\
\text { más cercanos como «mis amigos», «mis } \\
\text { colegas», «mis compañeros de trabajo/ } \\
\text { estudio». }\end{array}$ & 12 \\
\hline
\end{tabular}

En la siguiente tabla (Tabla 5), aparecen los datos de la dimensión relacionada con los sentimientos negativos ante la adquisición de los logros de los demás. Se seleccio- naron 4 ítems con un valor mínimo de 0.75 . Resaltar el consenso encontrado en esta dimensión en general y en concreto en los Ítem 40 y 44. 
Tabla 5

Dimensión E: Sentimientos negativos ante la adquisición de logros de los demás

\begin{tabular}{|c|c|c|c|c|}
\hline N. ${ }^{\circ}$ ítem & Enunciado & $I_{i k}$ & Comentarios de los expertos & $\mathbf{N}$ \\
\hline ítem 40 & $\begin{array}{l}\text { Me molesta que los demás logren } \\
\text { alguna cosa que yo quiero }\end{array}$ & 1.00 & & 12 \\
\hline ítem 44 & $\begin{array}{l}\text { Me da rabia que los demás consigan } \\
\text { cosas que llevo tiempo intentando } \\
\text { conseguir }\end{array}$ & 1.00 & & 12 \\
\hline ítem 34 & $\begin{array}{l}\text { Me siento triste cuando me doy } \\
\text { cuenta de que los otros consiguen } \\
\text { cosas que yo quiero }\end{array}$ & 0.75 & Identificación de autoestima & 12 \\
\hline ítem 35 & $\begin{array}{l}\text { Me enfado cuando alguien obtiene } \\
\text { mejores resultados. }\end{array}$ & 0.75 & $\begin{array}{l}\text { Identificación de un sentimiento de } \\
\text { culpabilidad. }\end{array}$ & 12 \\
\hline ítem 39 & $\begin{array}{l}\text { Cuando a alguien le ocurre algo } \\
\text { importante siento pena de que no me } \\
\text { haya ocurrido a mí }\end{array}$ & 0.25 & $\begin{array}{l}\text { Clarificar a qué se refiere con «algo } \\
\text { importante». También es algo importante } \\
\text { la muerte de un ser querido o ser } \\
\text { diagnosticado de una grave enfermedad y } \\
\text { ello no se suele desear ni envidiar. Por eso, } \\
\text { tal vez sea mejor colocar «algo positivo». }\end{array}$ & 12 \\
\hline ítem 48 & $\begin{array}{l}\text { Me siento muy bien si un compañero } \\
\text { logra mejores resultados que yo }\end{array}$ & -0.17 & & 12 \\
\hline
\end{tabular}

Por último, comentar que en la dimensión F solamente fue seleccionado un ítem (el número 5), pero con un consenso absoluto.

Tabla 6

Dimensión F: Relación con el envidiado

\begin{tabular}{|c|c|c|c|c|}
\hline N. ${ }^{\circ}$ ítem & Enunciado & $\mathrm{I}_{\mathrm{ik}}$ & Comentarios de los expertos & $\mathbf{N}$ \\
\hline ítem 5 & $\begin{array}{l}\text { Siento desprecio hacia las personas } \\
\text { que poseen las cosas que yo quiero }\end{array}$ & 1.00 & & 12 \\
\hline ítem 30 & $\begin{array}{l}\text { Intento que mis compañeros no sepan } \\
\text { las cosas que deseo y que no tengo }\end{array}$ & 0.00 & & 12 \\
\hline ítem 25 & $\begin{array}{l}\text { No quiero que los demás conozcan mis } \\
\text { defectos }\end{array}$ & -0.25 & & 12 \\
\hline ítem 27 & $\begin{array}{l}\text { Me gusta participar en proyectos y } \\
\text { actividades con las personas mejores } \\
\text { que yo. }\end{array}$ & -0.33 & & 12 \\
\hline ítem 10 & $\begin{array}{l}\text { Me llevo muy bien con las personas } \\
\text { que tienen éxito en el trabajo (estudio) } \\
\text { o en sus relaciones. }\end{array}$ & -0.42 & & 12 \\
\hline ítem 20 & $\begin{array}{l}\text { Soy muy buen amigo de los que } \\
\text { consiguen las cosas que yo quiero }\end{array}$ & -0.42 & $\begin{array}{l}\text { No me gusta la redacción... «Mantengo } \\
\text { la amistad con las personas que han } \\
\text { alcanzado éxitos que yo deseo» }\end{array}$ & 12 \\
\hline ítem 15 & $\begin{array}{l}\text { Me gustan las personas que son } \\
\text { mejores que yo en algo }\end{array}$ & -0.50 & & 12 \\
\hline
\end{tabular}


Resaltar que en los comentarios de los jueces aparecen posibles relaciones de los ítems con otros sentimientos y/o atributos psicológicos como son: la autoestima, la culpabilidad, la deseabilidad social, la comparación social... etc.

\section{Discusión}

En primer lugar, reseñar que la participación de expertos en la evaluación y/o medida, provenientes tanto de la universidad como de la asistencia psicológica implica un valor añadido a la validación de contenido realizada, ya que no se circunscribe solamente a uno de los posibles ámbitos de aplicación del instrumento resultante. El juicio de expertos realizado reduce el pool inicial de items, quedando éste formalmente constituido por 24 ítems. Tal como proponen Smith y Kim (2007), en los ítems del pool inicial no aparece la envidia explícitamente, lo que conlleva que los expertos identifiquen en sus comentarios los aspectos constituyentes de la misma.

A continuación se analiza el contenido de las diferentes dimensiones teóricas de la propuesta inicial descritos por Castilla del Pino (2000) con respecto a los ítems seleccionados.

Entre las dimensiones teóricas se observa la existencia de dos de ellas relacionadas con sentimientos negativos: una cuando se elogia a alguien que se admira (Dimension A) y otra ante los logros de los demás (Dimension E), ambas se encuentran representada en el cuestionario final por dos y cuatro ítems respectivamente con un grado alto de consenso $\left(\mathrm{I}_{\mathrm{ik}} \geq\right.$ 0.75). Estos ítems se identifican con el episodio emocional de resentimiento global (Parrot y Smith, 1993); Milfont y Gouveia (2009) lo incluyen en el componente depresivo de la envidia, nunca debemos olvidar que estos sentimientos se encuentran relacionados con la percepción de injusticia (Smith y Kim, 2007). De los ítems propuestos, relacionados con los pensamientos de injusticia ante los logros de los demás (Dimension D), la mayor parte han tenido un nivel adecuado de congruencia, lo que resulta indicativo de la adecuación de los ítems propuestos con esta dimensión teórica.
En la dimensión B de la envidia relacionada con la degradación de la posición social o imagen del envidiado, se consideran adecuados 8 ítems, la mayor parte de ellos con un muy alto nivel de congruencia, la importancia de la correspondencia entre esta dimensión con la envidia viene reflejada porque los ítems constituyentes de la misma se relacionan con el sentimiento de hostilidad presente en la envidia. (Smith y Kim, 2007). Igualmente el ítem seleccionado en la dimensión $\mathrm{F}$, indicativa de la relación con el envidiado, se puede considerar constituyente del sentimiento de hostilidad a pesar del nivel de consenso encontrado $\left(\mathrm{I}_{\mathrm{ik}}=1\right)$.

En la dimensión $\mathrm{C}$ referente al deseo de posesiones de los demás, encontramos que 4 ítems con una congruencia en el punto de corte $\left(\left(\mathrm{I}_{\mathrm{ik}}=0.50\right)\right.$ se han constituido como parte del cuestionario. Resaltar que siendo este componente esencial en la gestación de la envidia (Etxebarria, 2008; Riedl, 2005; Smith y Kim, 2007; Tangney y Salovey, 2010) debe constituir una parte fundamental para la construcción de un cuestionario que nos permita evaluarla tal como nos indican Carrasco et al (2004) al identificar que uno de los dos factores constituyentes de la envidia es el deseo por lo ajeno, estando todas las demás dimensiones teóricas propuestas inicialmente reflejadas en el factor relativo a la reacción emocional.

En resumen, se observa que todas las dimensiones propuestas por Castilla Del Pino (2000), se encuentran representadas, aunque por desigual número de ítems. La dimensión relativa a sentimientos negativos cuando se elogia a alguien admirado, se encuentra representada por dos ítems y la dimensión referente a la relación con la persona envidiada está representada por un solo ítem; en el extremo opuesto se encuentra la dimensión relacionada con la degradación de la persona envidiada, que está representada por ocho ítems. El resto de dimensiones están representadas: desear las cosas de los demás, los pensamientos de injusticia y sentimientos ante la adquisición de logros, en la versión final con cuatro o cinco ítems cada una.

La reducción de un $50 \%$ del pool inicial de ítems, conlleva que este cuestionario se com- 
pare respecto al realizado por Carrasco et al. (2004) en su estudio sobre envidia infantil, y así se puede considerar que en la versión final para la población adulta se encuentran sus equivalentes en 18 ítems; ya que seis ítems no aparecen en la versión infantil, estos ítems distintivos son el 8 "si un compañero tiene éxito suelo decir que ha sido suerte», el 11 "me siento mal cuando alguien reconoce las cualidades y lo bueno de mis compañeros», el 16 "me molesta que mis compañeros reciban elogios como yo querría para mí», el 19 "nadie debería ser mejor que yo», el 32 "cuando un compañero obtiene buenos resultados no ha sido por motivos propios» y el 43 «me gustaría que todos mis compañeros quisieran estar más conmigo que con los otros». Destacar que como se puede observar, los ítems 11 y 16 se encontraban relacionados con la dimensión teórica relativa a sentimientos negativos cuando se elogia a alguien admirado, por lo que en la versión final del CEA se encuentren representadas todas las dimensiones teóricas propuestas por Castilla Del Pino (2000), lo que en principio hace que se diferencie del CEI ya que en el mismo no aparece representada esta dimensión. La inclusión de los ítems 8 y 32 relativos a la degradación de la persona envidiada, acarrea que esta dimensión conste de un mayor número de ítems, tal como sucedía en el CEI.

Mencionar que solamente tres ítems de la escala de niños no se encuentran representados en esta versión para adultos. A pesar de estas diferencias se puede considerar la equiparación entre ambos, ya que el CEI contiene el $85,71 \%$ de los ítems constituyentes de la escala adaptada a la población adulta.

Como conclusión de este estudio señalar que se ha creado un Cuestionario para la evaluación de la envidia en adultos (CEA) compuesto de 24 ítems que son representativos de dicho constructo. Dicho cuestionario es una adaptación de la versión infantil, siendo su contenido equivalente.

Este trabajo es el primer eslabón del proceso de validación encaminado a la obtención de evidencias de validez de constructo y de criterio de la envidia, así que una vez obtenidos datos sobre la relevancia y representatividad de los ítems utilizados en el cuestionario, se deberá proceder al estudio de las propiedades psicométricas del mismo, que permita una mejor depuración de ítems para finalmente abordar el estudio de su dimensionalidad y de su relación con otros conceptos que se consideren relevantes.

\section{Referencias}

Anderson, M. K. (2011). Concretization and envy. Canadian Journal of Psychoanalysis/ Revue Canadienne de Psychanalyse, 19(1), 125-131.

Bediou, B., Mohri, C., Lack, J. y Sander D. (2011). Effects of outcomes and random arbitration on emotions in a competitive gambling task. Front Psychology, 2, 213.

Bonovitz, C. (2010). Comparative perspectives on envy: A reconsideration of its developmental origins. Contemporary Psychoanalysis, 46(3), 423-438.

Carrasco, M. A., Gonzalez-Calderon, M. J. y Del Barrio, M. J. (2004). Evaluación de la envidia infantil: Construcción de un instrumento autoinformado [Evaluation of Childhood envy: Building a self-report instrument]. RIDEP, 18(2), 9-27.

Coricelli, G. y Rustichini, A. (2010). Counterfactual thinking and emotions: regret and envy learning. Philosophical Transactions of the Royal Society B: Biological Sciences, 365(1538), 241-247.

Chóliz, M. y Gómez, C. (2002). Emociones sociales II (enamoramiento, celos, envidia y empatía). En F. Palmero, E. G. Fernández-Abascal, F. Martínez y M. Chóliz (Eds.), Psicología de la Motivación y Emoción [Psychology of Motivation and Emotion] (pp. 395-418). Madrid, España: McGraw-Hill.

Crusius, J. y Mussweiler, T. (2012). When people want what others have: The impulsive side of envious desire. Emotion, 12(1), 142-153.

Debbané, E. (2011). Envy and its relation to destructiveness. Canadian Journal of Psychoanalysis/ Revue Canadienne de Psychanalyse, 19(1), 108-124.

Dvash J., Gilam G., Ben-Ze'ev A., Hendler T. y Shamay-Tsoory S. G. (2010). The envious brain: 
the neural basis of social comparison. Human Brain Mapp. 31(11), 1741-1750.

Etchegoyen, R. H. y Nemas, C. R. (2003). Salieri's dilemma: a counterpoint between envy and appreciation. International Journal of Psychoanalysis, 84(1), 45-58.

Etxebarria, I. (2008). Emociones sociales: culpa, vergüenza, orgullo, envidia y celos. En P. Palmero y F. Martínez Sánchez (Coords.), Motivación y emoción [Motivation and Emotion] (pp. 275314). Madrid, España: McGraw-Hill.

Fernández-Abascal, E. G., Jiménez, M. P. y Martín, M. D. (2007). Emoción y Motivación. La adaptación humana [Emotion and Motivation. Human adaptation]. Madrid, España: Centro de Estudios Ramón Areces.

Fernández-Abascal, E. G. y Palmero, F. (1999). Emociones y salud [Emotions and Health]. Barcelona, España: Ariel.

Fiske, S. T. (2011). Envy up, scorn down: How status divides us. NY, EEUU: Russell Sage Foundation.

González, M. A., Carrasco, M. J. y del Barrio M. J. (2011). Estudio sobre la Red Nomológica de la Envidia en Población Infanto-juvenil [Study on the nomological network Envy Child and Adolescent Population]. Revista Latinoamericana de Psicología. 43, 45-58.

Heikkinen, E. e Isola, A. (2004). Student nurses' experiences and perceptions of envy in one nurse education environment in Finland. Nurse Education Today, 24(3), 160-168.

Heikkinen, E., Latvala, E. e Isola, A. (2003). Envy in a nurse education community. International Journal of Nurse Studies, 40(3), 259-268.

Hill S. E., DelPriore D. J. y Vaughan P. W. (2011). The cognitive consequences of envy: attention, memory, and self-regulatory depletion. Journal of Personality Social Psychology, 101(4), 653-66.

Izquierdo, C. y Johnson, A. (2004). Desire, envy and punishment: a Matsigenka emotion schema in illness narratives and folk stories. Culture, Medicine and Psychiatry, 31(4), 419-444.

Kragl, J. y Schmid, J. (2009). The impact of envy on relational employment contracts. Journal of Economic Behavior \& Organization, 72(2), 766-779.

Krajbich, I., Adolphs, R., Tranel, D., Denburg, N. L. y Camerer C. F. (2009). Economic games quantify diminished sense of guilt in patients with damage to the prefrontal cortex. Journal of Neuroscience, 29(7), 2188-2192.

Krizan, Z. y Johar, O. (2012). Envy Divides the Two Faces of Narcissism. Journal of Personality, 80(5), 1415-1451

Laverde-Rubio, E. (2004). Envy: one or many? International Journal of Psychoanalysis, 85(2), 401-418.

León, R. (2002). Un estudio acerca de la envidia en los centros laborales en el Perú [A study of envy in the workplace in Peru]. Persona, 5, 147-165.

Milfont, T. y Gouveia, V. (2009). A Capital Sin: Dispositional Envy and its Relations to Wellbeing. Revista Interamericana de Psicología/Interamerican Journal of Psychology, 43(3), 547-551.

McNamee, M. (2010). Schadenfreude in sports: Envy, justice and self-esteem. En M. McNamee (Ed.), The ethics of sports: A reader (pp. 327339). New York, NY, EEUU: Routledge/Taylor \& Francis Group.

Reidl, L. M. (2005). Celos y envidia: emociones humanas. México: UNAM postgrado.

Rodríguez, P. M., Parrott, W. G. y Hurtado, A. (2010). I fear your envy, I rejoice in your coveting: on the ambivalent experience of being envied by others. Journal of Personality Social Psychology, 99(5), 842-854.

Rosenberger, J. W. (2005). Envy, shame, and sadism. Journal American Academy of Psychoanalysis and Dynamic Psychiatry, 33(3), 465-489.

Sawada, M. (2010). Developmental features of envy. Japanese Psychological Review, 53(1), 110-123.

Shamay-Tsoory, S. G., Tibi-Elhanany, Y. y Aharon-Peretz J. (2007). The green-eyed monster and malicious joy: the neuroanatomical bases of envy and gloating (schadenfreude). Brain, 130(6), 1663-1678.

Shamay-Tsoory, S. G., Fischer M., Dvash J., Harari H., Perach-Bloom N. y Levkovitz, Y. (2009). Intranasal administration of oxytocin increases envy and schadenfreude (gloating). Biological Psychiatry, 66(9), 864-870.

Shoshani, M., Shoshani, B., Kella, R. y Becker M. (2011). Green eyes, crows, and scorpions: Envy in the contexts of neediness, separateness, and narcissism. Psychoanalytic Psychology. Advance online publication. 
Smith, R. H. y Kim, S. H. (2007). Comprehending envy. Psychological Bulletin, 133(1), 46-64.

Tai, K., Narayanan, J. y McAllister, D. J. (2012). Envy as pain: Rethinking the nature of envy and its implications for employees and organizations. The Academy of Management Review, 37(1), 107-129.

Tangney, J. P. y Salovey, P. (2010). Emotions of the imperiled ego: Shame, guilt, jealousy, and envy. En J. E. Maddux y J. P. Tangney (Eds.), Social psychological foundations of clinical psychology (pp. 245-271). NY, EEUU: Guilford Press.

Thompson, E. H. y Hampton, J. A. (2011). The effect of relationship status on communicating emotions through touch. Cogition and Emotion, 25(2), 295-306.

Van de Ven, N., Zeelenberg, M. y Pieters, R. (2009). Leveling up and down: the experiences of benign and malicious envy. Emotion, 9(3), 419-429.
Van de Ven, N., Zeelenberg, M. y Pieters, R. (2011). Why envy outperforms admiration. Personality and Social Psychology Bulletin, 37(6), 784-795.

Van de Ven, N., Zeelenberg, M. y Pieters, R. (2010). Warding off the evil eye: when the fear of being envied increases prosocial behavior. Psychological Science, 21(11), 1671-1682.

Van Dijk, W., Ouwerkerk, J. W., Wesseling, Y. M. y van Koningsbruggen, G. M. (2011). Towards understanding pleasure at the misfortunes of others: the impact of self-evaluation threat on schadenfreude. Cognition and Emotion, 25(2), 360-368.

West, M. (2010). Envy and difference. Journal of Analytical Psychology, 55(4), 459-484.

Winarick, K. (2010). Thoughts on greed and envy. Journal of the American Academy of Psychoanalysis, 70(4), 317-327. 